\title{
Modelling the magnetic field caused by a dc-electrified railway with linearly changing leakage currents
}

\author{
Risto Pirjola \\ Finnish Meteorological Institute, P. O. Box 503, FI-00101 Helsinki, Finland \\ (Received December 17, 2010; Revised May 4, 2011; Accepted June 20, 2011; Online published January 26, 2012)
}

\begin{abstract}
Magnetic fields produced by dc railways can disturb operations at geomagnetic observatories. These magnetic fields consist of two parts: the ideal "full-loop" field due to the traction current in an overhead wire and in the rails, and the "leakage" field due to currents leaking from the track into the ground. Pirjola et al. (2007) present formulas with numerical examples for the magnetic field assuming a constant leakage current density between the train and the feeding substation. Lowes (2009) considers this assumption questionable. In this paper, we derive exact formulas for the magnetic field assuming that the leakage current density changes linearly being largest at the train and decreasing to zero at the substation. The Earth is considered layered. Computer codes enable calculations of the magnetic fields due to any number and configuration of the train-substation pairs at any points of observation. Numerical examples show that the value of $10 \mathrm{pT}$ can easily be exceeded. An important conclusion is that the magnetic fields in the cases of a linearly changing and a constant leakage current density do not differ much. Particular attention is paid to the temporal behaviour of the magnetic field when one or two trains travel along a railway.
\end{abstract}

Key words: Geomagnetic observatory, dc train, dc magnetic field, current leakage in the ground.

\section{Introduction}

Quasi-dc electric currents produce magnetic fields that can seriously disturb recordings and operations at geomagnetic observatories, at which the maximum allowable noise levels are about $10^{-2} \mathrm{nT}=10 \mathrm{pT}$ today. This maximum value is based on private discussions with representatives of the Ottawa Geomagnetic Observatory, Canada, and its choice is also argued by Pirjola et al. (2007). Consequently, advance calculations and estimations of expected magnetic fields at geomagnetic observatories due to possible dc equipment and devices being in the planning stage are very important. Problematic magnetic fields are created especially by dc-electrified railways (e.g., Yanagihara, 1977; Georgescu et al., 2002; Pirjola et al., 2007; Lowes, 2009; and references therein). There are historical examples of the need to close a geomagnetic observatory and move it to a more distant location because a dc-electrified railway or tramway makes the operation impossible (e.g. Nevanlinna, 2004). Ishikawa et al. (2007) discuss the noise due to dc trains in geoelectric field data in connection with the development of earthquake prediction techniques.

In principle, the traction current needed to move a train should flow in a closed circuit formed by an overhead feeding wire and a return path along the rails. Due to the proximity of the wire and the equal, but opposite, return current, the magnetic field should decrease sharply with the distance from the railway. In practice, however, the rails may have

Copyright (c) The Society of Geomagnetism and Earth, Planetary and Space Sciences (SGEPSS); The Seismological Society of Japan; The Volcanological Society of Japan; The Geodetic Society of Japan; The Japanese Society for Planetary Sciences; TERRAPUB.

doi:10.5047/eps.2011.06.032 electric contacts with the ground enabling a current leakage into the Earth. Thus, the return current does not cancel the magnetic effect of the current in the traction wire. Moreover, the leakage current may flow to large distances in the ground.

Based on the model presented by Georgescu et al. (2002) for studies of magnetic fields caused by a dc-electrified railway and by utilising the Biot-Savart law, Pirjola et al. (2007) derive the formulas for the ideal full-loop magnetic field, i.e. no leakage, and for the leakage magnetic field, and the total field is naturally the sum of these two contributions. It should be emphasised that the formulas are exact including somewhat complicated algebraic manipulations. This is, however, justifiably criticised by Frank Lowes in his impressive and significant paper (Lowes, 2009) because the geometry and the physical interpretations are obscured by the algebra. On the other hand, since exact closed-form solutions exist that are easy to handle in computer calculations, they are worth being used in numerical modelling of the magnetic fields in question.

Another criticism that Lowes (2009) presents concerns the assumption made both by Georgescu et al. (2002) and by Pirjola et al. (2007) that the leakage current $(=j d x)$ is constant along the track, i.e. the leakage current density per unit length $(=j)$ is the same at each infinitesimal section $[x, x+d x]$ between the substation $(x=0)$ and the train $(x=L)$. On page viii of Lowes (2009), the constantleakage approximation is called "a completely unrealistic situation". Lowes proves this statement by careful investigations of the track-to-soil voltages and the associated leakage currents in different cases.

To get an idea of the effect of spatial variations of the 
leakage current density $j$, it is assumed in this paper that $j$ is a linear function of the position coordinate $x$ between the substation and the train. Referring to section 4 of Lowes (2009), a linear variation is not an exact form of the leakage current but, according to his section 3, a linear function is a reasonable approximation. Similarly to Georgescu et al. (2002) and by Pirjola et al. (2007), we assume that the current, which leaks into the ground everywhere along the track between the train and the substation, returns to the system at the substation. Thus, the situation considered in this paper corresponds to figure 3(b) of Lowes (2009), i.e. the leakage current is zero at the substation and increases linearly reaching its maximum at the train.

Another reason for the assumption of $j$ being a linear function of $x$ is that, similarly to the case of a constant leakage, the magnetic field turns out to have exact closed-form expressions in this case as well. In this paper, we indicate that a linearly changing leakage model results in a larger magnetic field than a constant leakage model. However, the difference between the two cases is small, and so in practice, both models can be applied to estimating magnetic disturbances produced by dc-electrified railways.

In Section 2 of this paper, we present the theoretical model including a linearly changing leakage current density and give the formulas for the magnetic field. Section 3 is devoted to numerical calculations, and Section 4 provides concluding remarks.

It is necessary to emphasise that the numerical values used in the calculations are chosen to be comparable with Pirjola et al. (2007), even if they do not correspond to modern railways precisely.

\section{Theoretical Model}

Similarly to Pirjola et al. (2007), we consider a rectangular loop consisting of a train-substation pair and of the track section and the overhead traction wire between the train and the substation. Let the distance from the substation to the train and the height of the overhead wire be $L$ and $h$, respectively. The rectangle thus has two horizontal sides of the length $L$ and two vertical sides of the length $h$. One horizontal side lies at the Earth's surface and the other at the height $h$. The train is fed by a dc traction current $J_{1}$ flowing along the overhead wire from the substation to the train.

A right-handed Cartesian $x y z$ coordinate system is used where the $x$ axis is parallel to the above-mentioned rectangular loop with the substation and the train lying at $x=0$ and $x=L$, respectively. The plane of the rectangular loop and Earth's surface define the $x z$ plane and the $x y$ plane, respectively. The $z$ axis points downwards.

In an ideal situation, the traction current $J_{1}$ returns from the train to the substation along the rails but in practice a certain amount, denoted by $J_{0}$, of the current leaks into the earth and takes a path determined by the ground conductivity before going back to the system at the substation, which is assumed to be the location of the return of all the current to the system. The leakage at a given infinitesimal section $[x, x+d x](0 \leq x \leq L)$ is given by $j(x) d x$ where $j=j(x)$ is the leakage current density per unit length. It should be noted that we neglect the track extensions be- yond the substation and beyond the train (at $x<0$ and $x>L$ ). This assumption includes an approximation (see Lowes, 2009). However, to be consistent and comparable with the study presented by Pirjola et al. (2007), the assumption of neglecting the extensions is also made in this paper. Furthermore, Georgescu et al. (2002) support the neglect, too. At this point, it is also necessary to emphasise that a theoretical model always necessarily suffers from different shortcomings, so that it is not appropriate to try to look at every minor detail when a model is considered as a whole.

Mathematically and physically the situation discussed in this paper can be described by thinking that in addition to the ideal rectangular full loop we have a set of elementary leakage loops. Each of them carries a current $j(x) d x$ and consists of a part from $x^{\prime}=0$ to $x^{\prime}=x$ along the rails and of a ground return path from $x^{\prime}=x$ to $x^{\prime}=0$. Along the rails, this current is opposite to the full-loop current $J_{1}$, i.e. it flows to the positive $x$ direction. This means that the leakage current flowing towards the train at a point $x$ $(0 \leq x \leq L)$ is

$$
J=J(x)=\int_{x}^{L} j\left(x^{\prime}\right) d x^{\prime}
$$

Thus the real current flowing along the rails towards the substation at this point is

$$
J_{\text {rail }}=J_{\text {rail }}(x)=J_{1}-J(x)=J_{1}-\int_{x}^{L} j\left(x^{\prime}\right) d x^{\prime}
$$

We define the total leakage current to be equal to $J(x=0)$ and denote it by $J_{0}$, i.e.

$$
J_{0}=\int_{0}^{L} j\left(x^{\prime}\right) d x^{\prime}
$$

Pirjola et al. (2007) considered $j\left(x^{\prime}\right)$ to be independent of $x^{\prime}$, and so from Eq. (3), $j=J_{0} / L$. Now, we assume that $j\left(x^{\prime}\right)$ is a linear function of $x^{\prime}$, i.e.

$$
j=j\left(x^{\prime}\right)=k x^{\prime}
$$

where $k$ is a constant, which can be determined in terms of $J_{0}$ and $L$ by using Eq. (3) leading to

$$
k=\frac{2 J_{0}}{L^{2}}
$$

As concerns the full loop, the resulting magnetic field is, of course, exactly the same as that given by equations (4)(10) of Pirjola et al. (2007). Thus we can directly adopt the following formulas for the contribution of the full loop to the total magnetic field

$$
\begin{aligned}
B_{x}^{F}= & B_{2} \frac{y}{\sqrt{y^{2}+x^{2}}}-B_{4} \frac{y}{\sqrt{y^{2}+(L-x)^{2}}} \\
B_{y}^{F}= & -B_{2} \frac{x}{\sqrt{y^{2}+x^{2}}}-B_{3} \frac{h}{\sqrt{y^{2}+h^{2}}} \\
& -B_{4} \frac{L-x}{\sqrt{y^{2}+(L-x)^{2}}} \\
B_{z}^{F}= & -B_{1}+B_{3} \frac{y}{\sqrt{y^{2}+h^{2}}}
\end{aligned}
$$




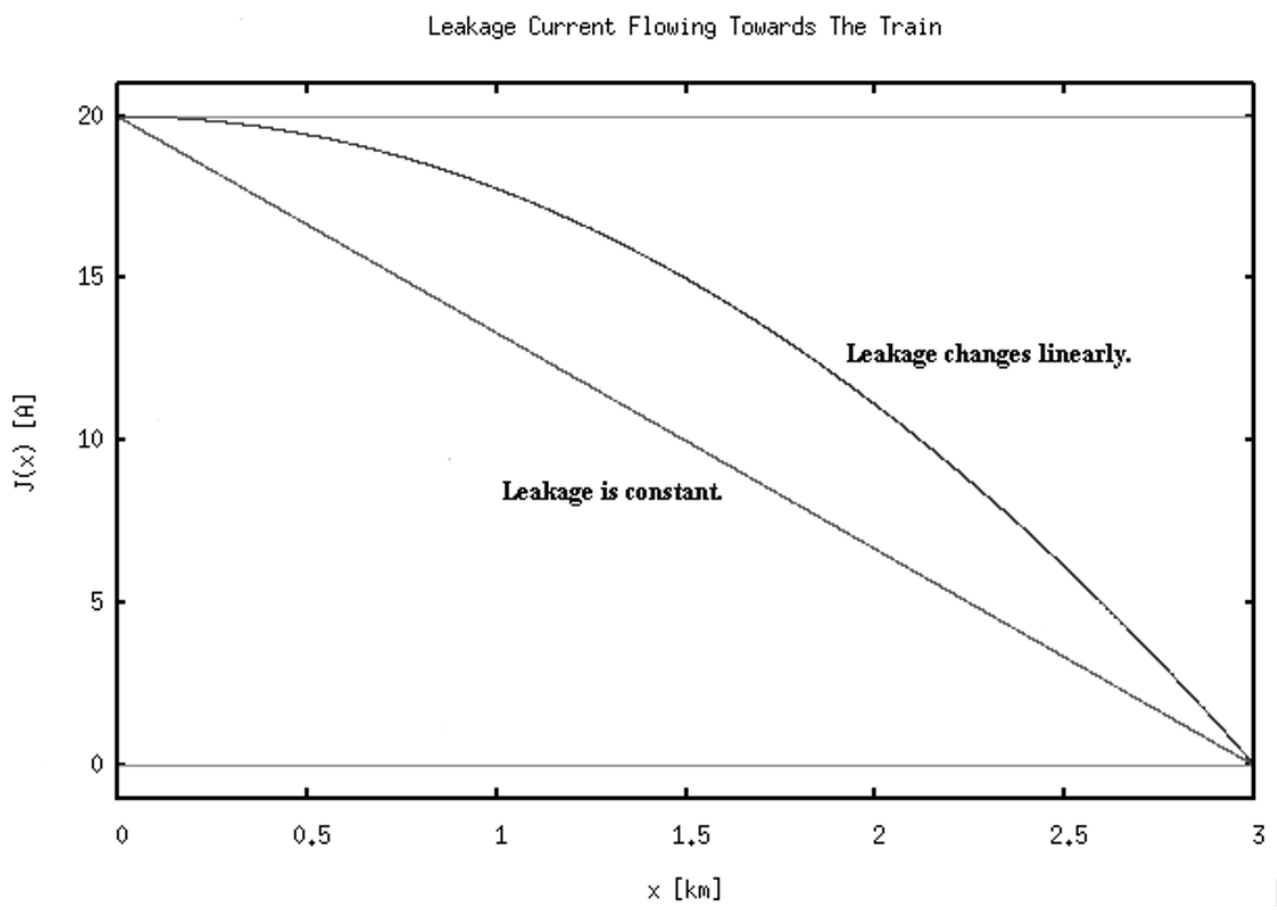

Fig. 1. Leakage current $J=J(x)$ given by Eq. (1) between the substation at $x=0$ and the train at $x=L=3 \mathrm{~km}$. The total leakage current expressed by Eq. (3) equals $J_{0}=20 \mathrm{~A}$. The lower (red) line and the upper (blue) curve correspond to a constant and to a linearly increasing leakage current density $j=j(x)$ per unit length between $x=0$ and $x=L$, respectively.

where

$$
\begin{aligned}
& B_{1}=\frac{\mu_{0} J_{1}}{4 \pi y}\left(\frac{L-x}{\sqrt{y^{2}+(L-x)^{2}}}+\frac{x}{\sqrt{y^{2}+x^{2}}}\right) \\
& B_{2}=\frac{\mu_{0} J_{1}}{4 \pi \sqrt{y^{2}+x^{2}}} \frac{h}{\sqrt{y^{2}+x^{2}+h^{2}}} \\
& B_{3}=\frac{\mu_{0} J_{1}}{4 \pi \sqrt{y^{2}+h^{2}}}\left(\frac{x}{\sqrt{y^{2}+x^{2}+h^{2}}}\right. \\
& \left.B_{4}=\frac{L-x}{4 \pi \sqrt{y^{2}+(L-x)^{2}}} \frac{\mu_{0} J_{1}}{\sqrt{y^{2}+(L-x)^{2}+h^{2}}}\right)
\end{aligned}
$$

These $B_{k}(k=1,2,3,4)$ quantities are the fields created by the four sides of the full loop. As usual, $\mu_{0}$ is the vacuum permeability $\left(=4 \pi \cdot 10^{-7} \frac{\mathrm{Vs}}{\mathrm{Am}}\right)$.

Similarly to Pirjola et al. (2007), we assume that the Earth is layered (and in the extreme case even uniform). Therefore the leakage currents flow radially symmetrically from the point of injection to all directions in the ground. Based on a modified version of the "Fukushima theorem" (Fukushima, 1976), such a current distribution creates exactly the same magnetic field at the Earth's surface as a semi-infinite vertical line current starting from the surface downwards. Thus the ground part of the elementary leakage loop that carries a current $j\left(x^{\prime}\right) d x^{\prime}$ can be replaced with a vertical upward current at $x=y=0,0 \leq z \leq \infty$ and a vertical downward current at $x=x^{\prime}, y=0,0 \leq z \leq \infty$. The horizontal parts of the leakage loops lie at $z=0$ and $z=\infty$, the latter of which does not contribute to the mag- netic field at the Earth's surface.

Figure 1 depicts the leakage current $J=J(x)$ defined by Eq. (1) between the substation located at $x=0$ and the train located at $x=L=3 \mathrm{~km}$. The total leakage current given by Eq. (3) equals $J_{0}=20 \mathrm{~A}$. The lower line (with $J(x)=J_{0}\left(1-\frac{x}{L}\right)$ ) and the upper curve (with $\left.J(x)=J_{0}\left(1-\frac{x^{2}}{L^{2}}\right)\right)$ correspond to a constant and to a linearly increasing leakage current density $j=j(x)$ per unit length between $x=0$ and $x=L$, respectively. The leakage current $J(x)$ gives the total amount of current in the elementary leakage loops whose width is larger than or equal to $x$. Therefore, since $J(x)$ in the linearly increasing case is always larger than in the constant case, larger elementary leakage currents are carried by wider loops in the former case. This implies that the magnetic fields are obviously larger in the linearly increasing case than in the constant case. The same conclusion can also be drawn directly from the growth of the leakage current density $j(x)$ with $x$ in the case of the linear increase. Next we derive exact formulas for the magnetic field components $B_{x}^{L}, B_{y}^{L}$ and $B_{z}^{L}$ produced by all elementary leakage loops, and the total field is the sum of the full and leakage contributions:

$$
B_{u}^{\mathrm{TOTAL}}=B_{u}^{F}+B_{u}^{L}
$$

where $u$ stands for $x, y$ or $z$.

The magnetic field due to an elementary leakage loop has exactly the same form as equations (11)-(13) of Pirjola et al. (2007), except that $j=j\left(x^{\prime}\right)=k x^{\prime}$ is a function of $x^{\prime}$, i.e.

$$
d B_{x}=-\frac{\mu_{0} k x^{\prime} d x^{\prime}}{4 \pi}\left(\frac{y}{y^{2}+\left(x^{\prime}-x\right)^{2}}-\frac{y}{y^{2}+x^{2}}\right)
$$




$$
\begin{aligned}
& d B_{y}=-\frac{\mu_{0} k x^{\prime} d x^{\prime}}{4 \pi}\left(\frac{x^{\prime}-x}{y^{2}+\left(x^{\prime}-x\right)^{2}}+\frac{x}{y^{2}+x^{2}}\right) \\
& d B_{z}=\frac{\mu_{0} k x^{\prime} d x^{\prime}}{4 \pi y}\left(\frac{x^{\prime}-x}{\sqrt{y^{2}+\left(x^{\prime}-x\right)^{2}}}+\frac{x}{y^{2}+x^{2}}\right)
\end{aligned}
$$

The integration of Eqs. (14)-(16) from $x^{\prime}=0$ to $x^{\prime}=L$ leads to the following formulas for the leakage contribution to the magnetic field

$$
\begin{aligned}
& B_{x}^{L}=\int_{0}^{L} d B_{x}=-g y\left(C_{1}+C_{2}\right) \\
& B_{y}^{L}=\int_{0}^{L} d B_{y}=-g\left(F_{1}+F_{2}\right) \\
& B_{z}^{L}=\int_{0}^{L} d B_{z}=\frac{g}{y}\left(P_{1}+P_{2}\right)
\end{aligned}
$$

where

$$
\begin{aligned}
g & =\frac{\mu_{0} k}{4 \pi}=\frac{\mu_{0} J_{0}}{2 \pi L^{2}} \\
C_{1} & =\int_{0}^{L} \frac{x^{\prime}}{y^{2}+\left(x^{\prime}-x\right)^{2}} d x^{\prime} \\
C_{2} & =-\frac{1}{y^{2}+x^{2}} \int_{0}^{L} x^{\prime} d x^{\prime} \\
F_{1} & =\int_{0}^{L} \frac{\left(x^{\prime}-x\right) x^{\prime}}{y^{2}+\left(x^{\prime}-x\right)^{2}} d x^{\prime} \\
F_{2} & =\frac{x}{y^{2}+x^{2}} \int_{0}^{L} x^{\prime} d x^{\prime} \\
P_{1} & =\int_{0}^{L} \frac{\left(x^{\prime}-x\right) x^{\prime}}{\sqrt{y^{2}+\left(x^{\prime}-x\right)^{2}}} d x^{\prime} \\
P_{2} & =\frac{x}{\sqrt{y^{2}+x^{2}}} \int_{0}^{L} x^{\prime} d x^{\prime}
\end{aligned}
$$

The terms $C_{2}, F_{2}$ and $P_{2}$ can be directly calculated because the integral in them simply gets the value $\frac{L^{2}}{2}$. The terms $C_{1}$, $F_{1}$ and $P_{1}$ require more work but they can also expressed in terms of elementary functions in closed forms as follows

$$
\begin{aligned}
C_{1}= & \ln \left(\sqrt{\frac{y^{2}+(L-x)^{2}}{y^{2}+x^{2}}}\right) \\
& +\frac{x}{y}\left(\arctan \left(\frac{L-x}{y}\right)+\arctan \left(\frac{x}{y}\right)\right) \\
F_{1}= & L+x \ln \left(\sqrt{\frac{y^{2}+(L-x)^{2}}{y^{2}+x^{2}}}\right) \\
& -y\left(\arctan \left(\frac{L-x}{y}\right)+\arctan \left(\frac{x}{y}\right)\right) \\
P_{1}= & \left.L \sqrt{y^{2}+(L-x)^{2}}\right) \\
& -\frac{y|y|}{2}\left(\operatorname { l n } \left[\left(\frac{L-x}{y}+\sqrt{\frac{(L-x)^{2}}{y^{2}}+1}\right)\right.\right. \\
& \left.\times\left(\frac{x}{y}+\sqrt{\frac{x^{2}}{y^{2}}+1}\right)\right]
\end{aligned}
$$

$$
\left.+\frac{L-x}{y} \sqrt{\frac{(L-x)^{2}}{y^{2}}+1}+\frac{x}{y} \sqrt{\frac{x^{2}}{y^{2}}+1}\right)
$$

We may thus finally write the formulas for the magnetic field components produced by the leakage currents

$$
\begin{aligned}
& B_{x}^{L}=-\frac{\mu_{0} J_{0} y}{2 \pi L^{2}}\left(\ln \left(\sqrt{\frac{y^{2}+(L-x)^{2}}{y^{2}+x^{2}}}\right)\right. \\
& +\frac{x}{y}\left(\arctan \left(\frac{L-x}{y}\right)+\arctan \left(\frac{x}{y}\right)\right) \\
& \left.-\frac{L^{2}}{2\left(y^{2}+x^{2}\right)}\right) \\
& B_{y}^{L}=-\frac{\mu_{0} J_{0}}{2 \pi L^{2}}\left(L+x \ln \left(\sqrt{\frac{y^{2}+(L-x)^{2}}{y^{2}+x^{2}}}\right)\right. \\
& -y\left(\arctan \left(\frac{L-x}{y}\right)+\arctan \left(\frac{x}{y}\right)\right) \\
& \left.+\frac{x L^{2}}{2\left(y^{2}+x^{2}\right)}\right) \\
& B_{z}^{L}=\frac{\mu_{0} J_{0}}{2 \pi y L^{2}}\left(L \sqrt{y^{2}+(L-x)^{2}}\right. \\
& -\frac{y|y|}{2}\left(\operatorname { l n } \left[\left(\frac{L-x}{y}+\sqrt{\frac{(L-x)^{2}}{y^{2}}+1}\right)\right.\right. \\
& \left.\times\left(\frac{x}{y}+\sqrt{\frac{x^{2}}{y^{2}}+1}\right)\right] \\
& \left.+\frac{L-x}{y} \sqrt{\frac{(L-x)^{2}}{y^{2}}+1}+\frac{x}{y} \sqrt{\frac{x^{2}}{y^{2}}+1}\right) \\
& \left.+\frac{x L^{2}}{2 \sqrt{y^{2}+x^{2}}}\right)
\end{aligned}
$$

The three components of the magnetic field due to the trainsubstation system with a linearly changing leakage current are now obtained from Eq. (13) with Eqs. (6)-(8) and (30)(32). It seems evident that, in practice, the total leakage current depends on the distance $L$ between the train and the substation. Thus, it might be more reasonable to express $J_{0}$ as $J_{0}=\frac{k L^{2}}{2}$ in Eqs. (30)-(32), i.e. in terms of $k$ and $L$, in accordance with Eq. (5). The constant $k$ is clearly proportional to the traction current (but not necessarily linearly proportional). Similarly, in the corresponding equations presented by Pirjola et al. (2007), it would obviously be more reasonable to use the quantity $j$ instead of $J_{0}(=j L)$.

The $x y z$ coordinate system used above is fixed to the particular train-substation pair. Thus when investigating the magnetic field produced by several trains operating simultaneously we actually have many $x y z$ systems, in which only the $z$ axes are the same, i.e. downward vertical and having the point $z=0$ at the Earth's surface. The components of the total magnetic field at a given point of observation have to be expressed in a single fixed coordinate 
Absolute Value of the Magnetic Field Caused by an Electrified Railway

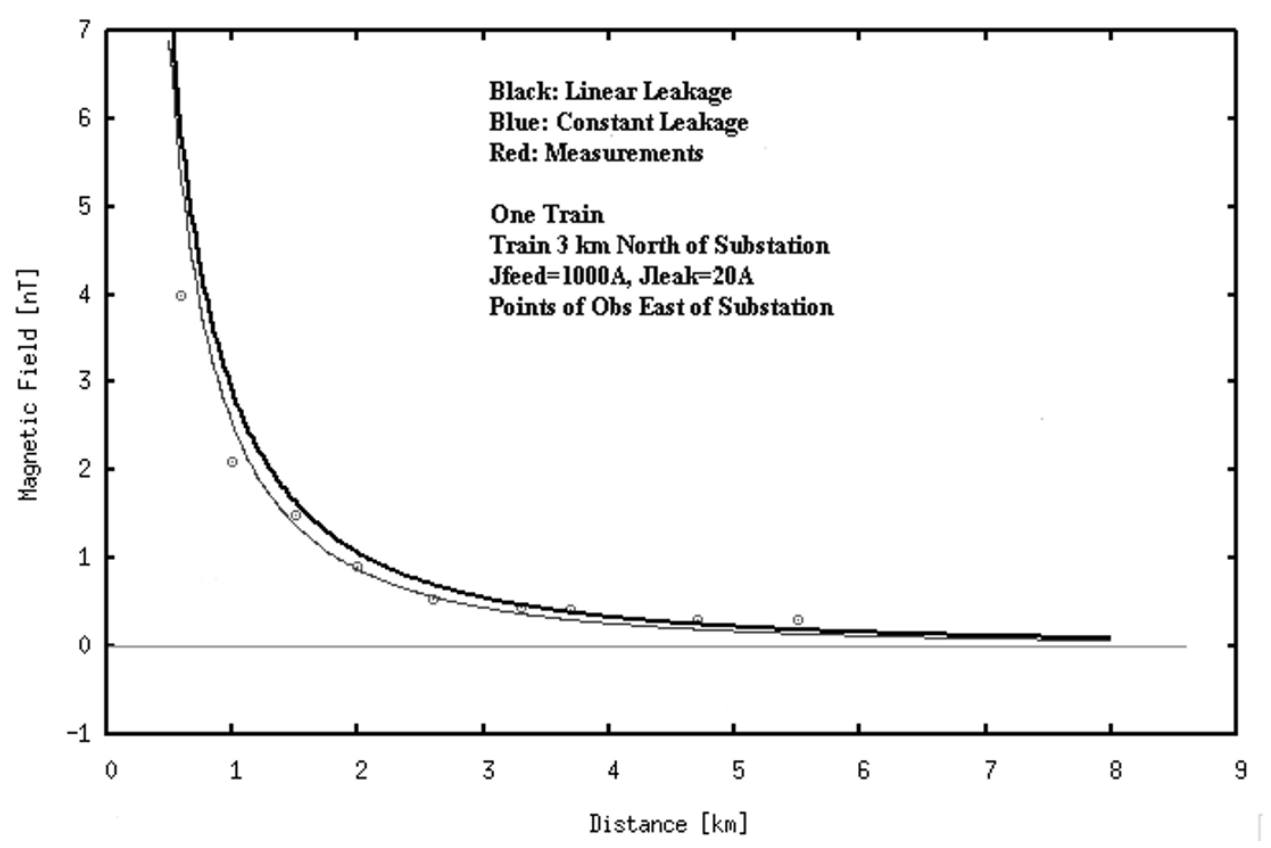

Fig. 2. Absolute values of the magnetic field due to a south-north dc-electrified railway. The thicker upper (black) curve and the thinner lower (blue) curve refer to a linearly changing and to a constant leakage current density, respectively. The horizontal axis gives the distance along a line towards the east from the substation. Additional details of the calculations are given in the text. The small (red) circles show measured data in Calgary, Canada, in March 2006 (cf. Pirjola et al., 2007).

system, and our choice is to use, besides the $z$ component, the (geographical) north and east components. To achieve this, information of the geographical direction of the line between each train and the corresponding substation(s) is needed. A practical way is to express all locations in terms of their geographical latitudes and longitudes. In practice, the areas to be considered are small enough to enable simple "flat-Earth" relations between latitudes and longitudes and Cartesian coordinates.

Based on the formulas presented above, "Octave/MatLab" program codes have been prepared to enable numerical computations of the magnetic fields due to a dc-electrified railway, in which the train-substation pairs and the points of observation may have any number, configuration and parameter values.

\section{Numerical Results}

We now consider the situation that is associated with the measurements carried out in the vicinity of a (nearly) straight south-north dc-electrified railway in Calgary, Canada, in March 2006 (see Pirjola et al., 2007, section 4). Unfortunately, the configuration of the railway system in Calgary and the technical details including the values of the parameters associated with the operation of the system are unknown. Therefore theoretical modelling of the situation during the measurements is based on unconfirmed assumptions and conclusions. This emphasises the need for additional magnetic field measurements in Calgary or somewhere else to confirm the validity of the theoretical modelling (or to reveal its shortcomings).

Similarly to figure 7 of Pirjola et al. (2007), we assume that there is only one train fed by a current of $J_{1}=1000 \mathrm{~A}$ from a substation located at a distance of $3 \mathrm{~km}$ south of the train, i.e. $L=3 \mathrm{~km}$. The total leakage current $J_{0}$ and the height of the feeding wire $h$ are $20 \mathrm{~A}$ and $5 \mathrm{~m}$, respectively. Thus, a constant leakage current density is $j=J_{0} / L=6.7 \mathrm{~mA} / \mathrm{m}$. For a linearly changing leakage current density, Eq. (5) implies that the constant $k$ equals $k=2 J_{0} / L^{2}=0.0044 \mathrm{~mA} / \mathrm{m}^{2}$, so that the leakage current density varies from $13.3 \mathrm{~mA} / \mathrm{m}$ at the train $(x=L)$ to zero at the substation $(x=0)$.

The upper (black) and lower (blue) curves in Fig. 2 show the absolute values of the magnetic field for a linearly changing and a constant leakage, respectively. The horizontal axis gives the distance along a line towards the east from the substation. The small (red) circles show the measured data in Calgary. In fact, the lower curve, together with the measurements, re-produces the information included in figure 7 of Pirjola et al. (2007). We see that the choice between a linearly changing and a constant leakage does not imply a big difference in the magnetic field, and both models agree well with measurements. However, the lack of knowledge of technical parameters associated with the Calgary railway has to be kept in mind. The measured data shown in Fig. 2 are concluded from abrupt jumps in the time-dependent signals that otherwise follow the natural variations of the geomagnetic field. Besides, a scaling of the magnetic field is performed based on a reference site (see Pirjola et al., 2007).

The observation from Fig. 2 that a linearly changing leakage results in a larger magnetic field than a constant leakage can be understood by noting that, in the linear case, the elementary leakage loops with the largest sizes also carry the highest currents, as explained in Section 2. Assuming a lin- 


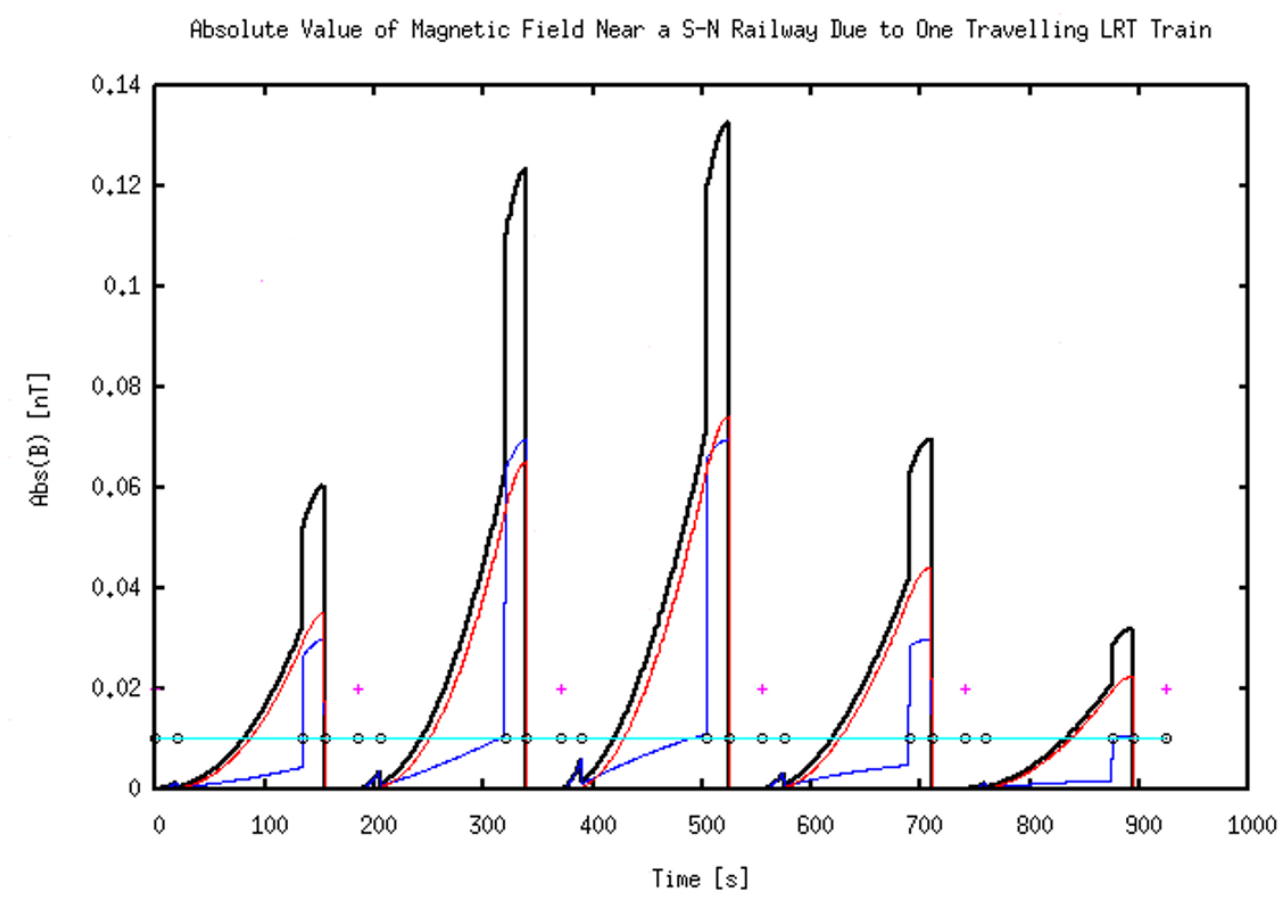

Fig. 3. Absolute values of the full-loop (lowermost, blue), leakage (middle, red) and total (uppermost, black) magnetic fields at a point of observation located at $5 \mathrm{~km}$ east of a south-north railway as functions of time produced by a train travelling from south to north. The leakage current density is assumed to be constant. The maximum allowable noise level at a geomagnetic observatory (10 pT) is shown by the horizontal (light blue) line. The small (black) circles and the small (red) crosses are related to the schedule of the train. Additional details of the calculation are given in the text.

early changing leakage improves the agreement with measured magnetic data at larger distances whereas the agreement becomes slightly worse at smaller distances. Considering disturbing magnetic fields to be accepted at an observatory, small distances are, however, less important because there the magnetic fields are definitely too high and thus need not be investigated precisely.

Next we consider the time dependence of the magnetic field at a point of observation when one train travels or two trains travel along the railway. Such calculations have been performed for the planned "Light Rail Transit (LRT)" system in Ottawa, Canada, but in this paper, we simply continue considering a straight south-north railway. It is assumed to consist of five sections between substations, each of them $3 \mathrm{~km}$ in length, so that the total length of the railway is $15 \mathrm{~km}$ and the number of substations is six. Let the point of observation be located at $5 \mathrm{~km}$ east of the third substation from south.

The trains are always assumed to get the traction current from the nearest substation south of the train by feeding wires at the height of $6 \mathrm{~m}$. The trains are assumed to stop at every substation (and nowhere else) with the following schedule:

- Acceleration from zero to the (maximum) speed of $v_{\max }=80 \mathrm{~km} / \mathrm{h}$ during $T_{a}=20 \mathrm{~s}$.

- Constant speed of $v_{\max }=80 \mathrm{~km} / \mathrm{h}$.

- Deceleration from $v_{\max }=80 \mathrm{~km} / \mathrm{h}$ to zero during $T_{d}=20 \mathrm{~s}$.

- Stop at the substation for $T_{s}=30 \mathrm{~s}$.

During the acceleration deceleration phases, the traction current is set to $J_{1}=3000 \mathrm{~A}$, and during the constant speed phase to $J_{1}=500 \mathrm{~A}$. (These values are based on private discussions in North America in 2010.)

The choice of an appropriate value for the leakage current density seems to involve a lot of discrepancies. In the above calculation, we have the constant value of $6.7 \mathrm{~mA} / \mathrm{m}$. Some unpublished information indicates leakage current densities of $20 \mathrm{~mA} / 1000$ feet $\approx 0.067 \mathrm{~mA} / \mathrm{m}$, i.e. a difference of a factor of 100. In the computations presented now, we use the value of $950 \mathrm{~mA} / 300 \mathrm{~m}=3.17 \mathrm{~mA} / \mathrm{m}(\approx 950 \mathrm{~mA} / 1000$ feet) for a constant leakage current density. (It is based on a private discussion in North America in 2010.) When the leakage current density is constant, the total leakage current at a track length $L$ is $j L$. To make the cases of a constant and of a linearly changing leakage current density comparable, we want that, in the case of a linearly changing leakage current density, the total leakage current at a track length $L$ is also $j L$, which implies from Eq. (5) that $k=$ $2 j / L$ with $j=950 \mathrm{~mA} / 300 \mathrm{~m}$.

For a constant leakage current density, the lowermost (blue), middle (red) and uppermost (black) curves in Fig. 3 present the absolute values of the full-loop magnetic field $\left(\mathbf{B}^{F}\right)$, of the leakage magnetic field $\left(\mathbf{B}^{L}\right)$ and of the total magnetic field $\left(\mathbf{B}^{\text {TOTAL }}=\mathbf{B}^{F}+\mathbf{B}^{L}\right)$ at the point of observation as functions of time, respectively. (Note that since we consider the absolute values of three-dimensional vectors the sum of the blue and red curves is generally not equal to the black curve.) Figure 4 corresponds to Fig. 3 but a linearly changing leakage current density is assumed. Similarly to Fig. 2, Figs. 3 and 4 show that a linearly changing leakage current density leads to somewhat higher magnetic fields at the point of observation, whose explanation results from high currents in large elementary leakage loops in the 
Absolute Value of Magnetic Field Near a S-N Railway Due to One Travelling LRT Train

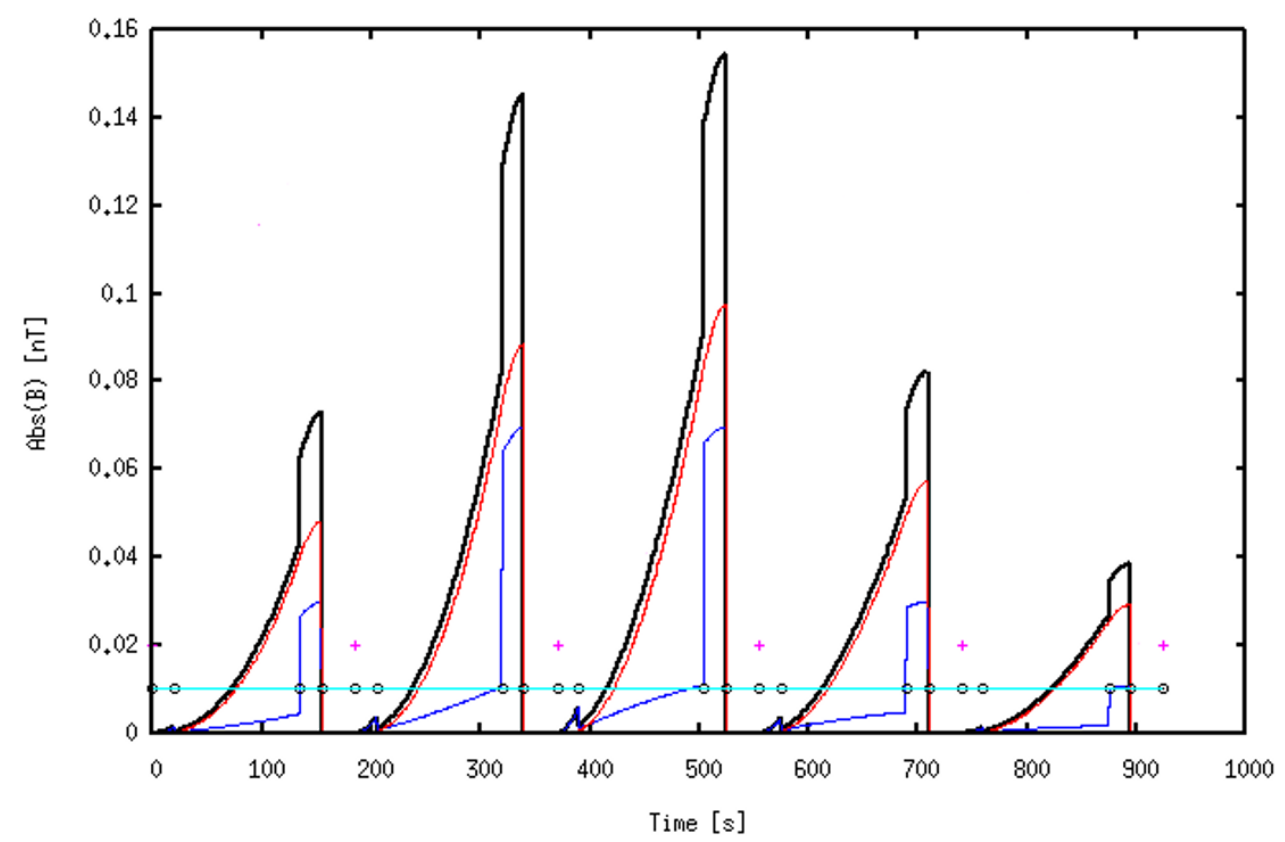

Fig. 4. Similar to Fig. 3, but a linearly changing leakage current density is assumed. Additional details of the calculation are given in the text.

Absolute Value of Magnetic Field Near a S-N Railway Due to Two Travelling LRT Trains

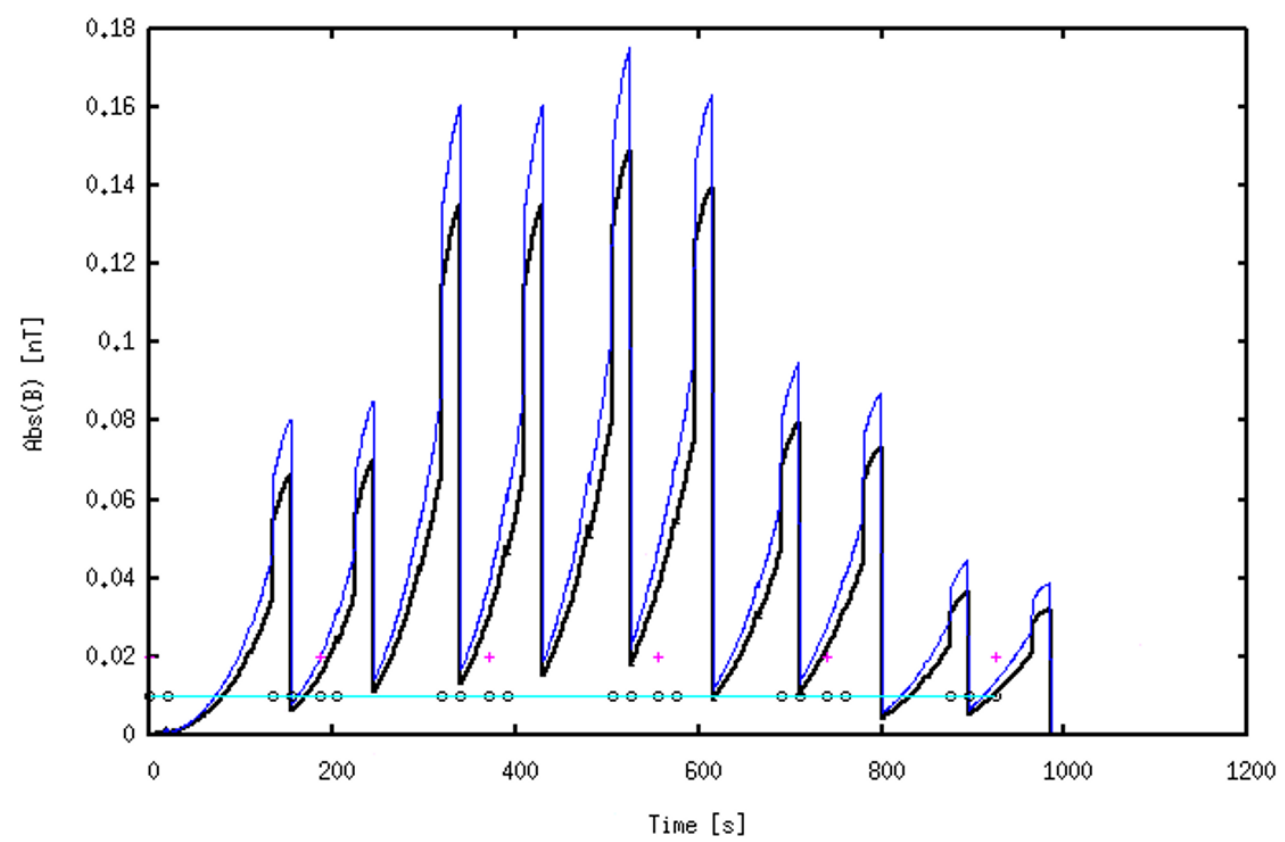

Fig. 5. Absolute value of the (total) magnetic field a point of observation located at $5 \mathrm{~km}$ east of a south-north railway as a function of time produced by two identical trains travelling from south to north at 90 -s separation. The thicker lower (black) and the thinner upper (blue) curves correspond to a constant and a linearly changing leakage current density, respectively. The maximum allowable noise level at a geomagnetic observatory (10 pT) is shown by the horizontal (light blue) line. The small (black) circles and the small (red) crosses are related to the schedule of the first train. Additional details of the calculations are given in the text.

case of a linearly changing leakage current density, as mentioned above. Both in Fig. 3 and in Fig. 4, the maximum allowable noise level at a geomagnetic observatory $(=$ the horizontal line at $10^{-2} \mathrm{nT}$ ) is clearly exceeded during most of the travel time of the train. We also see that the leakage magnetic field is usually larger than the full-loop magnetic field.

So far we have only considered the magnetic field created by one train but in practice there are usually several trains travelling simultaneously. In Fig. 5, it is assumed that a train is followed by another identically operating train after $90 \mathrm{~s}$. The lower (black) and the upper (blue) curves depict 
the absolute value of the magnetic field at the point of observation for a constant and a linearly changing leakage current density, respectively. In agreement with Figs. 3 and 4 , the linearly changing leakage results in a larger magnetic field. The magnetic field is above the maximum allowable noise level practically all the time.

An important conclusion obtained from Figs. 3, 4 and 5 and already drawn from Fig. 2 is that the magnetic fields in the cases of a linearly changing and a constant leakage current density do not differ much from each other. Consequently, both leakage current models can be equally utilised for practical estimation of magnetic fields due to dc railways.

In the numerical computations discussed in this section, we only consider the absolute values of the magnetic field. The theory and formulas presented in Section 2 would naturally enable studies of individual components of the magnetic field separately. However, regarding practical investigations of disturbances at observatories due to magnetic fields, the absolute value is the quantity of importance. Moreover, the absolute value is larger than the components and thus represents the worst case.

\section{Concluding Remarks}

Railways operating by dc electricity create disturbing magnetic fields, which may cause problems at geomagnetic observatories. Of particular significance in this respect is the amount of currents leaking from the track into the ground. As a supplement to the paper by Pirjola et al. (2007), exact formulas for the magnetic field due to a dcelectrified railway are presented in this paper by assuming that the leakage current density per unit length decreases linearly from the train to the substation. Pirjola et al. (2007) consider a spatially constant leakage current density, which is an assumption criticised by Lowes (2009).

The theoretical model and formulas introduced in this paper, as well as those discussed by Pirjola et al. (2007), together with the pertinent computer programs, allow the investigation of the magnetic fields created by any number and configuration of train-substation pairs with any parameter values at any points of observation. Numerical examples presented in this paper demonstrate the usefulness of the model as a practical tool for estimating magnetic disturbances due to dc-electrified railways. They also indicate that the maximum allowable noise level set to $10 \mathrm{pT}$ nowadays is easily exceeded at a nearby geomagnetic observatory.

It is indicated in this paper that a linearly changing leakage current density results in a larger magnetic field than a constant leakage, which is explained by noting that, in the linear case, the elementary leakage loops with the largest sizes also carry the highest currents. The difference between the cases of a linearly changing and a constant leakage current density is, however, so small that it would certainly vanish in errors due to many approximations included in theoretical models. This means that, in practical estimations of magnetic fields caused by dc-electrified railways, both models are equally applicable.

In the computations considered in this paper, the traction current is assumed to be taken to trains from only one station in a railway section. However, it is important to realise that, in practice, a train may also be fed from both ends of a section (Ishikawa et al., 2007; private discussions in Ottawa, Canada, in 2010). Our numerical calculations not presented in this paper, whose principal objective is to introduce the model and formulas associated with a linearly changing leakage current density and to compare the models of linearly changing and constant leakage currents, show that a two-end feeding significantly reduces the magnetic fields as compared to a one-end feeding. Thus the question about the feeding system plays an important role when magnetic disturbances due to a dc-electrified railway are considered. There are also many other items in railway technology including the leakage currents that have to be known exactly to obtain correct estimates of the resulting magnetic fields. All this emphasises the need for interaction between geophysical scientists and railway engineers.

Acknowledgments. The author wishes to thank the personnel of the Geomagnetic Laboratory of the Natural Resources Canada in Ottawa, Canada, for introducing the topic of magnetic fields due to dc-electrified railways and for excellent collaboration on the subject. Thanks go especially to David Boteler, Lorne McKee, Larry Newitt and Benoit St-Louis, and to Peter Fernberg and Gerrit Jansen van Beek, who carried out the measurements in Calgary in 2006. The author also wants to acknowledge Frank Lowes (Newcastle University, UK) for many interesting and very useful email discussions about modelling dc railways. Finally, the author is obliged to the two unknown reviewers of the manuscript for many constructive and helpful comments and suggestions.

\section{References}

Fukushima, N., Generalized theorem for no ground magnetic effect of vertical currents connected with Pedersen currents in the uniformconductivity ionosphere, Rep. Ionos. Space Res. Jpn., 30(1/2), 35-40, 1976.

Georgescu, P., J.-L. Le Mouel, and M. Mandea, Electric and magnetic perturbations generated by d.c. electric railway, Geofizica, Bucuresti, Romania, 40, 69-82, 2002.

Ishikawa, H., K. Hattori, I. Takahashi, Y. Noda, T. Nagao, and N. Isezaki, Effect of noise from DC-driven trains to geoelectrical potential difference and its reduction in Hakuba area, Japan, IEEJ Trans. Fund. Mater., 127(1), 41-47, (Extended Summary on p. 3), 2007.

Lowes, F. J., DC railways and the magnetic fields they produce-the geomagnetic context, Earth Planets Space, 61, i-xv, 2009.

Nevanlinna, H., Results of the Helsinki magnetic observatory 1844-1912, Ann. Geophys., 22, 1-15, 2004.

Pirjola, R., L. Newitt, D. Boteler, L. Trichtchenko, P. Fernberg, L. McKee, D. Danskin, and G. Jansen van Beek, Modelling the disturbance caused by a dc-electrified railway to geomagnetic measurements, Earth Planets Space, 59(8), 943-949, 2007.

Yanagihara, K., Magnetic field disturbance produced by electric railway, Mem. Kakioka Magn. Obs. suppl. 7, Geophys. Mag., 38(1), 17-35, 1977.

R. Pirjola (e-mail: risto.pirjola@fmi.fi) 